\title{
Metabolic and Lipidomic Profiling of Vegetable Juices Fermented with Various Probiotics
}

\author{
Hyuk-Jin Chung ${ }^{1,2}$, Hwanhui Lee ${ }^{1}$, Guknam Na ${ }^{2}$, Heechul Jung ${ }^{2}$, Dong-Gun Kim ${ }^{2}$, \\ Sang-Ick Shin ${ }^{2}$, Seong-Eun Jung ${ }^{2}$, Il-dong Choi ${ }^{2}$, Jae-Hwan Lee ${ }^{2}$, Jae-Hun Sim ${ }^{2}$ and \\ Hyung-Kyoon Choi ${ }^{1, *}$ \\ 1 College of Pharmacy, Chung-Ang University, Seoul 06974, Korea; hjchung0812@gmail.com (H.-J.C.); \\ hwanhui56@gmail.com (H.L.) \\ 2 Korea Yakult Co., Ltd., Yongin 17086, Korea; biongn@re.yakult.co.kr (G.N.); yk58jhc@re.yakult.co.kr (H.J.); \\ kimdg@re.yakult.co.kr (D.-G.K.); muse123@re.yakult.co.kr (S.-I.S.); krus00@re.yakult.co.kr (S.-E.J.); \\ cid1010@re.yakult.co.kr (I.-d.C.); jaehwan@re.yakult.co.kr (J.-H.L.); jhsim@re.yakult.co.kr (J.-H.S.) \\ * Correspondence: hykychoi@cau.ac.kr ; Tel.: +82-2-820-5605; Fax: 82-2-812-3921
}

Received: 3 March 2020; Accepted: 2 May 2020; Published: 6 May 2020

\begin{abstract}
Fermented vegetable juices have gained attention due to their various beneficial effects on human health. In this study, we employed gas chromatography-mass spectrometry, direct infusion-mass spectrometry, and liquid chromatography-mass spectrometry to identify useful metabolites, lipids, and carotenoids in vegetable juice (VJ) fermented with Lactobacillus plantarum HY7712, Lactobacillus plantarum HY7715, Lactobacillus helveticus HY7801, and Bifidobacterium animalis ssp. lactis HY8002. A total of 41 metabolites, 24 lipids, and 4 carotenoids were detected in the fermented and non-fermented VJ (control). The lycopene, $\alpha$-carotene, and $\beta$-carotene levels were higher in VJ fermented with L. plantarum strains (HY7712 and HY7715) than in the control. Proline content was also elevated in VJ fermented with HY7715. Uracil, succinic acid, and $\alpha$-carotene concentration was increased in VJ fermented with HY7801, while glycine and lycopene levels were raised in VJ fermented with HY8002. This study confirmed that each probiotic strain has distinctive characteristics and produces unique changes to metabolic profiles of VJ during fermentation. Our results suggest that probiotic-fermented VJ is a promising functional beverage that contains more beneficial metabolites and carotenoids than commercial non-fermented VJ.
\end{abstract}

Keywords: vegetable juice fermented with probiotics; Lactobacillus; Bifidobacterium; metabolic profiling; lipidomic profiling

\section{Introduction}

The World Health Organization (WHO) defines probiotics as living microorganisms that, when consumed at a sufficient level, promote host health [1]. Representatively, Lactobacillus acidophilus, B. lactis, Enterococcus faecalis, Enterococcus faecium, Lactococcus lactis, and Streptococcus thermophilus are probiotic strains that produce lactic acid. Bacillus and Saccharomyces strains, which do not produce lactic acid, are also considered probiotics [2]. Traditional probiotic-fermented foods, such as yogurt, cheese, miso, and kimchi, are commonly consumed for their health benefits [3]. Consuming probiotics is clinically proven to reduce symptoms related to imbalanced gut microbiota, abnormal immune system response related to cold and influenza, cardiovascular disease, and gastrointestinal discomfort [4].

Vegetable juices (VJs) have gained widespread popularity as an alternative to raw vegetables and fruits to supply micronutrients, phenolic compounds, carotenoids, and fiber [5,6]. A diet rich in vegetables may reduce the risk of cardiovascular disease, protect from oxidative stress, and prevent some types of cancer [7-9]. Multiple studies have suggested the beneficial effects of fermented 
vegetables and VJs. Lactobacillus, Lactococcus, and Enterococcus strains are present in traditional fermented health foods, such as sauerkraut, pickled cucumbers, and kimchi [10], and consuming fermented Asian vegetables is an easy way to boost probiotic intake [11]. Strawberry, onion, and tomato juices fermented by Lactobacillus, Lactococcus, Leuconostoc, and Saccharomyces display antioxidant capacity compared to non-fermented vegetable juices [12]. The antioxidant properties of orange and carrot juices are enhanced following fermentation by two Bifidobacterium strains [6]. In addition, kale juice fermented by different Lactobacillus strains is abundant in calcium, phosphorus, and magnesium, and those fermented with L. acidophilus IFO 3025 and L. brevis FSB-1 display improved nutritional and mineral composition, respectively [13]. The color and level of volatile compounds in fruit and VJs are also known to change after fermentation [12].

Metabolomics and lipidomics can be used to investigate the metabolic and lipidomic changes in vegetables and VJ during fermentation. Recently, it was reported that nuclear magnetic resonance spectrometry (NMR) and liquid chromatography-mass spectrometry (LC-MS) can rapidly discriminate the metabolic profiles of vegetable juice and medium fermented with different Lactobacillus strains [14,15]. This suggests that different lactic acid bacteria have unique characteristics that influence the types of metabolites produced, and multivariate data analysis can be used to assess the metabolic changes following fermentation. Filannino et al., who analyzed vegetable and fruit juices using LC and gas chromatography-mass spectrometry (GC-MS), found that the levels of malic acid, branched-chain amino acids, and gamma-aminobutyric acid were altered following fermentation [16]. Tomita et al., who subjected 45 metabolites and 62 volatile compounds in sunki (a fermented pickle from Japan) to NMR and GC-MS analysis, reported that acetic acid concentration was positively correlated with $\mathrm{pH}$ and negatively correlated with lactate and ethanol levels [17]. In another study, metabolites in fermented ginseng extracts were analyzed using GC-MS and an electronic tongue [18]. Ginseng extracts fermented by four different starter cultures could be distinguished according to their sugar and organic acid content, as well as their taste.

In this study, we hypothesized that each probiotic strain is able to generate distinct metabolic and lipidomic profiles during fermentation of VJ. To test this hypothesis, we used GC-MS, direct infusion-mass spectrometry (DI-MS), and LC-MS to assess the relative levels of various metabolites and intact lipid species in VJs fermented by four different probiotic strains: Lactobacillus plantarum HY7712, Lactobacillus plantarum HY7715, Lactobacillus helveticus HY7801, and Bifidobacterium animalis ssp. lactis HY8002. Several beneficial metabolites and lipid species, including carotenoids, were discovered in fermented VJ, which could have practical implications for improving public health.

\section{Materials and Methods}

\subsection{Probiotic Cultures and Vegetable Juice Fermentation}

The VJ and fermented VJs were provided by Korea Yakult Co., Ltd. (Yongin, Korea). The juice consisted of tap water, 18-24\% organic carrot juice concentrate (Ernteband, Winnenden, Germany), 7-10\% organic tomato paste (Attianese, Naples, Italy), 1-3\% organic mixed VJ 1 (lettuce 41\%, celery 32\%, spinach 27\%) (MSC, Gyeongsangnam-do, Korea), 0.01-1\% organic broccoli juice (MSC, Gyeongsangnam-do, Korea), 0.01-1\% organic zucchini and pumpkin juice concentrate (Ernteband, Winnenden, Germany), and 0.01-1\% organic mixed VJ 2 (bok choy 22\%, tatsoi 21\%, lettuce 20\%, broccoli leaf $11 \%$, crown daisy $11 \%$, curled mustard leaf 8\%, chard 7\%) (MSC, Gyeongsangnam-do, Republic of Korea). The juice composition was based on "Haru Yache Original" from Korea Yakult Co., Ltd. Lactobacillus strains HY7712, HY7715, and HY7801, and Bifidobacterium HY8002, from the Korea Yakult Probiotics Library (Yongin, Korea), were cultured in De Man, Rogosa and Sharpe (MRS) media (BD Difco, Maryland, USA) before each strain $\left(>10^{7} \mathrm{CFU} / \mathrm{mL}\right.$ ) was inoculated into a sample of the sterilized VJ ( $\left.30 \mathrm{~min}, 10{ }^{\circ} \mathrm{C}\right)$. Non-inoculated VJ ( $\mathrm{pH} 4.8$ ) was considered as the control. The inoculated juices were fermented at $37^{\circ} \mathrm{C}$ for $24 \mathrm{~h}$ (The $24 \mathrm{~h}$-fermented VJs with HY7712: $1.5 \times 10^{9} \mathrm{CFU} / \mathrm{mL}$, pH 3.8; HY7715 : $1.0 \times 10^{9} \mathrm{CFU} / \mathrm{mL}, \mathrm{pH} 3.8 ; \mathrm{HY7801}: 1.1 \times 10^{8} \mathrm{CFU} / \mathrm{mL}$, $\mathrm{pH} 3.6 ; \mathrm{HY} 8002$ : $\left.7.9 \times 10^{8} \mathrm{CFU} / \mathrm{mL}, \mathrm{pH} 4.1\right)$, then stored at $-70{ }^{\circ} \mathrm{C}$ until analysis. 


\subsection{Comprehensive Metabolic Profiling Using GC-MS}

The fermented and non-fermented juices $(100 \mu \mathrm{L})$ were transferred to separate microfuge tubes (Eppendorf, Hamburg, Germany), and extracted with $1 \mathrm{~mL}$ of methanol (HPLC grade; Fisher Scientific, Pittsburgh, PA, USA). The samples were briefly vortexed and sonicated for $30 \mathrm{~min}$ at $40 \mathrm{kHz}$ to improve extraction yields [19]. After sonication, the samples were centrifuged at $1000 \times g$ for $3 \mathrm{~min}$ at $4{ }^{\circ} \mathrm{C}$, and the supernatant was filtered through a $0.45 \mu \mathrm{m}$ polytetrafluoroethylene (PTFE) syringe filter (Whatman, Maidstone, UK). Of this, $200 \mu \mathrm{L}$ were transferred to GC vials and dried under nitrogen for $20 \mathrm{~min}$. The derivatization and GC-MS analysis of each sample were conducted according to previously reported methods [20]. A split ratio of 1:15 was used, and the detector voltage was set to $1153 \mathrm{~V}$. The oven temperature was set at $60^{\circ} \mathrm{C}$ and programmed to increase to $185^{\circ} \mathrm{C}$ at $5^{\circ} \mathrm{C} / \mathrm{min}$ (hold time $3 \mathrm{~min}$ ), then to $205^{\circ} \mathrm{C}$ at $3{ }^{\circ} \mathrm{C} / \mathrm{min}$, and finally to $310^{\circ} \mathrm{C}$ at $5^{\circ} \mathrm{C} / \mathrm{min}$.

\subsection{Comprehensive Lipid Profiling Using DI-MS}

The fermented and non-fermented juices $(50 \mu \mathrm{L})$ were transferred to separate microfuge tubes, and intact lipid species were extracted using the modified Matyash methyl tert-butyl ether (MTBE) method [21,22]. Briefly, $1 \mathrm{~mL}$ of MTBE (Sigma-Aldrich, St. Louis, MO, USA), $300 \mu \mathrm{L}$ of methanol, and $10 \mu \mathrm{L}$ of phosphatidylethanolamine (PE) 17:0/17:0 as an internal standard were added and vortexed. The sample was incubated for $1 \mathrm{~h}$ with shaking at room temperature. Two hundred fifty microliters of water (HPLC grade, Fisher Scientific, Pittsburg, PA) was added to sample for phase separation, and then the mixture was centrifuged at $1000 \times g$ for $10 \mathrm{~min}$. The upper phase was collected and dried under nitrogen gas. The dried lipid extract was dissolved in $300 \mu \mathrm{L}$ of chloroform/methanol $(2: 1, v / v)$ solution. For DI-MS analysis, methanol/chloroform $(9: 1, v / v)$ containing $7.5 \mathrm{mM}$ ammonium acetate solution was added to each lipid extract. DI-MS analysis of each sample was performed as previously reported [22]. A linear ion-trap mass spectrometer (LTQ-XL, Thermo Fisher Scientific, San Jose, CA, USA) coupled with an automated nanoelectrospray system (Triversa NanoMate System, Advion Biosciences, Ithaca, NY, USA) was used in positive- and negative-ion modes. The lipid extract was analyzed in full scan mode for $2 \mathrm{~min}$, and the scan range was set at $m / z$ 400-1200 and 500-1300 in positive and negative mode, respectively. Mass spectra were acquired in both positive mode (capillary voltage of $45 \mathrm{~V}$, tube lens voltage of $95 \mathrm{~V}$ ) and negative mode (capillary voltage of $-45 \mathrm{~V}$, tube lens voltage of $-95 \mathrm{~V}$ ). Tendem MS spectra was obtained to pooled samples from each group to identify lipid species. Lipid species were identified by comparing LipidBlast database by Kind et al. [23]. In addition, the in-house MS/MS library and Lipidmaps database [24] were used for identification.

\subsection{Carotenoid Analysis Using LC-MS}

The fermented and non-fermented juices $(50 \mu \mathrm{L})$ were transferred to separate microfuge tubes and extracted with $360 \mu \mathrm{L}$ of acetone (HPLC grade; Burdick \& Jackson, Musketon, MI, USA) containing $0.1 \%$ butylated hydroxytoluene (BHT; Sigma-Aldrich, St. Louis, MO) and $540 \mu \mathrm{L}$ of hexane (HPLC grade, Burdick \& Jackson) containing $0.1 \%$ BHT. The mixture was briefly vortexed, sonicated for 5 min at $4{ }^{\circ} \mathrm{C}$, then centrifuged at $1000 \times \mathrm{g}$ for $10 \mathrm{~min}$ at $4{ }^{\circ} \mathrm{C}$. After centrifugation, the supernatant was collected into microfuge tubes, and the residue re-extracted with $360 \mu \mathrm{L}$ of acetone and $540 \mu \mathrm{L}$ of hexane. The mixture was vortexed, sonicated, and centrifuged as described above. The supernatant was collected into the microfuge tubes used for the first extraction, and $200 \mu \mathrm{L}$ of water (HPLC grade; Fisher Scientific) with $0.1 \%$ BHT was added for phase separation. The top phase was collected and filtered through a $0.2 \mu \mathrm{m}$ PTFE syringe filter (Whatman). The filtrate was transferred into an amber vial, and $2 \mu \mathrm{L}$ of $\beta$-apo-8'-carotenal was added (Sigma-Aldrich, St. Louis, MO, USA), with $100 \mu \mathrm{g} / \mathrm{mL}$ used as an internal standard. Each sample was dried under nitrogen for $20 \mathrm{~min}$ and resuspended in $100 \mu \mathrm{L}$ of acetonitrile (HPLC grade; Fisher Scientific) and methanol solution $(7: 3, v / v)$. 
To increase the stability of the carotenoid standard solutions, a $100 \mu \mathrm{g} / \mathrm{mL}$ stock solution was prepared in hexane with $0.1 \%$ BHT [25]. The standard mixture was prepared with $100 \mu \mathrm{g} / \mathrm{mL}$ each of $\alpha$-carotene, $\beta$-carotene, and lycopene. Lutein $(20 \mu \mathrm{g} / \mathrm{mL})$ was added to the stock solution before the mixture was dried under nitrogen, and then resuspended in $100 \mu \mathrm{L}$ of acetonitrile:methanol solution $(7: 3 v / v)$. LC-MS analysis of each sample was conducted as previously reported, using an Accela LC (Thermo Fisher Scientific, San Jose, CA, USA) equipped with a degasser, Accela 600 pump, linear ion-trap mass spectrometer (LTQ-XL, Thermo Fisher Scientific), and Accela AS autosampler [26]. A $1.9 \mu \mathrm{m}$ Hypersil Gold column (Part no. 25002-102130; $100 \mathrm{~mm} \times 2.1 \mathrm{~mm}$; Thermo Scientific, San Jose, CA, USA) was used, and the column oven temperature was $35^{\circ} \mathrm{C}$. The autosampler tray temperature was $10{ }^{\circ} \mathrm{C}$, and the elution flow rate was $300 \mu \mathrm{L} / \mathrm{min}$. Water with $0.1 \%$ formic acid and a mixture of acetonitrile, methanol, and MTBE (70:20:10) with $0.1 \%$ formic acid served as solvents $A$ and $B$, respectively. The gradient was set to $25 \%$ solvent $A$ and $75 \%$ solvent $B$ and maintained for $2 \mathrm{~min}$. At $5 \mathrm{~min}$, solvent $\mathrm{B}$ was increased to $98 \%$ and maintained for $17 \mathrm{~min}$. After each run, the equilibrium time was 3 min with $25 \%$ solvent $A$ and $75 \%$ solvent $B$. The carotenoids in the fermented and non-fermented juice were identified by comparing the retention times and MS/MS spectra with those of corresponding carotenoid standards. Additionally, the control and fermented juices were analyzed on the micro-LC-LTQ-Orbitrap-XL instrument (Thermo Fisher Scientific) to identify the carotenoids by means of exact mass measurements and isotope patterns.

\subsection{Statistical Analysis}

The GC-MS, DI-MS, and LC-MS data were collected in Microsoft Office Excel (version 2016; Microsoft, Redmond, WA, USA) and used for principal component analysis (PCA), partial least squares-discriminant analysis (PLS-DA), and pathway analysis. The differences in the relative levels of metabolites, lipids, and carotenoids were evaluated by Mann-Whitney test in SPSS software (version 23; IBM, Somers, NY, USA), and those with $p<0.05$ were considered statistically significant. For PCA and PLS-DA, all data were mean-centered and scaled to unit variance in SIMCA-P+ software (version 13.0; Umetrics, Umeå, Sweden). Pathway analysis was performed in the web-based software tool MetaboAnalyst (version 4.0) [27].

\section{Results}

\subsection{Identification and Quantification of Metabolites and Lipids in Fermented and Non-Fermented VJs Using} GC-MS, DI-MS, and LC-MS

Comprehensive GC-MS analysis of the fermented and non-fermented VJs identified 41 metabolites: 13 amino acids ( $\beta$-alanine, $\gamma$-aminobutanoic acid, alanine, asparagine, aspartic acid, glutamic acid, glycine, isoleucine, proline, pyroglutamic acid, serine, threonine, and valine), 4 fatty acids (1-monopalmitin, linoleic acid, palmitic acid, and stearic acid), 8 organic acids (acetic acid, citric acid, fumaric acid, lactic acid, malic acid, malonic acid, succinic acid, and tartaric acid), 7 sugars (fructose, galactose, glucose, glucose-6-phosphate, sedoheptulose, sucrose, and xylose), 2 sugar acids (glyceric acid and threonic acid), 5 sugar alcohols (erythritol, glycerol, mannitol, myo-inositol, and xylitol), phosphoric acid, and uracil (Table 1). The levels of lactic acid and succinic acid were significantly higher, whereas those of $\beta$-alanine, asparagine, aspartic acid, pyroglutamic acid, serine, linoleic acid, fumaric acid, malic acid, tartaric acid, glucose, glyceric acid, erythritol, and phosphoric acid were significantly lower in VJ fermented with any of the four probiotics than in the control. In VJ fermented with L. plantarum HY7712, the levels of one fatty acid (stearic acid), two organic acids (lactic acid and succinic acid), and one alcohol (glycerol) were significantly higher, whereas those of other 28 metabolites were significantly lower, than in the control. In VJ fermented with L. plantarum HY7715, the concentrations of one amino acid (proline), two organic acids (lactic acid, succinic acid) and one sugar alcohol (glycerol) were significantly higher, whereas those of 23 other metabolites were significantly lower than in the control. Compared to non-fermented juice, VJ fermented with L. helveticus HY7801 had a higher content of two organic acids (lactic acid and succinic acid) and uracil and a lower content of 26 other metabolites. In VJ fermented with B. lactis 
HY8002, the levels of one amino acid (glycine), two organic acids (lactic acid and succinic acid), two sugars (glucose-6-phosphate and xylose), and one sugar alcohol (glycerol) were significantly higher, whereas those of 13 other metabolites were significantly lower, than in the control.

DI-MS analysis detected the following intact lipid species in the fermented and non-fermented VJs: three monogalactosyldiacylglycerols (MGDG; 18:2/18:3, 18:2/18:2, and 18:1/18:2), three lysophosphatidylcholines (Lyso-PC; 18:2, 18:1, and 22:5), one phosphatidylcholine (PC; 18:2/18:2), one phosphatidylethanolamine (PE; 16:0/20:0), and five triacylglycerides (TG; 16:0/18:2/18:2, 18:2/18:2/18:3, 18:2/18:2/18:2, 18:1/18:2/18:2, and 18:1/18:1/18:2) in positive ion mode, and two phosphatidic acids (PA; 16:0/18:2 and 18:2/18:2), four phosphatidylethanolamines (PE; 16:0/18:2, 18:2/18:2, 18:1/18:2, and 18:0/18:2), one phosphatidylglycerol (PG; 16:0/18:2), two phosphatidylserines (PS; 18:2/20:0 and 18:2/22:0), and two phosphatidylinositols (PI; 16:0/18:2 and 16:0/18:1) in negative ion mode (Table 2). In VJ fermented with L. plantarum HY7712, the concentration of PE 18:2/18:2, PG 16:0/18:2, PS 18:2/22:0, and PI 16:0/18:1 was significantly higher, whereas that of PE 18:0/18:2 was significantly lower, than in the control. In VJ inoculated with L. plantarum HY7715, the levels of PE 18:2/18:2, PS 18:2/22:0, and PI 16:0/18:1 were significantly higher, while those of PE 16:0/18:2 and 18:0/18:2 were significantly lower, than in the control. In VJ fermented with L. helveticus HY7801, the levels of PE 18:2/18:2, PE 18:1/18:2, PG 16:0/18:2, PS 18:2/22:0, and PI 16:0/18:1 were significantly higher, while those of PE 18:0/18:2 and PS 18:2/20:0 were significantly lower, than in non-fermented juice. VJ fermented with B. lactis HY8002 had a significantly higher content of PE 18:2/18:2, PS 18:2/22:0, and PI 16:0/18:1 and a significantly lower content of Lyso-PC 18:2 and PE 18:0/18:2 than in the control.

Relative levels of carotenoids in the different VJ samples are listed in Table 3. The concentration of lycopene, $\alpha$-carotene, and $\beta$-carotene was significantly higher in VJ fermented with L. plantarum HY7712 and HY7715 than in the control. VJ fermented with L. helveticus HY7801 had lower levels of lutein and higher levels of $\alpha$-carotene, while VJ fermented with B. lactis HY8002 had significantly higher levels of lycopene, than in the control. 
Table 1. Relative levels of metabolites in the fermented and non-fermented VJs, as detected by GC-MS.

\begin{tabular}{|c|c|c|c|c|c|c|c|c|c|c|}
\hline No. & Compound & $m / z$ & $\begin{array}{c}\text { RT } \\
\text { (min) }\end{array}$ & $\begin{array}{l}\text { Fragmentation } \\
\text { Ions }(\mathrm{m} / \mathrm{z})\end{array}$ & TMS & Control & $\begin{array}{l}\text { L. plantarum } \\
\text { (HY7712) }\end{array}$ & $\begin{array}{l}\text { L. plantarum } \\
\text { (HY7715) }\end{array}$ & $\begin{array}{l}\text { L. helveticus } \\
\text { (HY7801) }\end{array}$ & B. lactis (HY8002) \\
\hline & Amino acids & & & & & & & & & \\
\hline 1 & $\beta$-alanine & 174 & 17.37 & $100, \mathbf{1 7 4}, 248,290$ & 3 & $0.352 \pm 0.032$ & ND & ND & $0.302 \pm 0.011^{+}$ & $0.328 \pm 0.032$ \\
\hline 2 & $\gamma$-aminobutanoic acid & 174 & 19.85 & $174,216,246,304$ & 3 & $83.630 \pm 2.806$ & $66.176 \pm 8.063 *$ & $84.661 \pm 1.777$ & $77.351 \pm 2.740^{+}$ & $81.219 \pm 6.131$ \\
\hline 3 & Alanine & 116 & 8.80 & 100, 116, 190, 218 & 2 & $50.422 \pm 1.816$ & $50.480 \pm 2.053$ & $50.026 \pm 5.861$ & $53.394 \pm 3.054$ & $40.845 \pm 24.358$ \\
\hline 4 & Asparagine & 116 & 23.13 & 116, 132, 188, 231 & 3 & $20.862 \pm 1.038$ & $17.253 \pm 1.558 *$ & $18.952 \pm 0.744^{\#}$ & $13.289 \pm 1.015^{+}$ & $16.524 \pm 2.890$ \\
\hline 5 & Aspartic acid & 232 & 19.68 & $100,202,218,232$ & 3 & $190.073 \pm 8.232$ & $142.789 \pm 2.909 *$ & $159.894 \pm 1.455^{\text {\# }}$ & $157.900 \pm 5.864^{+}$ & $122.123 \pm 15.734$ \\
\hline 6 & Glutamic acid & 246 & 22.06 & $128,156,246,348$ & 3 & $175.620 \pm 4.918$ & $152.488 \pm 7.547 *$ & $154.917 \pm 3.394 \#$ & $134.961 \pm 5.251^{+}$ & $163.626 \pm 19.308$ \\
\hline 7 & Glycine & 174 & 14.19 & $86, \mathbf{1 7 4}, 248,276$ & 2 & $3.261 \pm 0.147$ & $3.118 \pm 0.448$ & $2.453 \pm 0.061^{\#}$ & $2.163 \pm 0.071^{+}$ & $5.763 \pm 0.237^{\wedge}$ \\
\hline 8 & Isoleucine & 158 & 13.88 & $100, \mathbf{1 5 8}, 218,232$ & 2 & $6.115 \pm 0.241$ & $2.297 \pm 0.237 *$ & $1.711 \pm 0.171^{\#}$ & $2.861 \pm 0.080^{+}$ & $4.462 \pm 1.543$ \\
\hline 9 & Proline & 142 & 13.96 & $100, \mathbf{1 4 2}, 144,216$ & 2 & $4.192 \pm 0.234$ & $4.804 \pm 0.729$ & $6.941 \pm 0.784$ \# & $4.736 \pm 0.149^{+}$ & $3.890 \pm 3.297$ \\
\hline 10 & Pyroglutamic acid & 156 & 19.62 & $133, \mathbf{1 5 6}, 230,258$ & 2 & $562.497 \pm 15.928$ & $483.337 \pm 18.371$ * & $508.725 \pm 11.686^{\#}$ & $521.194 \pm 20.262^{+}$ & $526.650 \pm 16.360$ \\
\hline 11 & Serine & 204 & 15.68 & $100,188,204,218$ & 3 & $14.700 \pm 0.692$ & $1.474 \pm 0.232 *$ & $7.357 \pm 0.522 \#$ & $13.816 \pm 0.457^{+}$ & $10.529 \pm 3.635^{\wedge}$ \\
\hline 12 & Threonine & 218 & 16.32 & $101,117, \mathbf{2 1 8}, 291$ & 3 & $4.661 \pm 0.119$ & $1.712 \pm 0.157 *$ & $2.217 \pm 0.037 \#$ & $2.617 \pm 0.156^{+}$ & $4.863 \pm 1.161$ \\
\hline 13 & $\begin{array}{c}\text { Valine } \\
\text { Fatty acids }\end{array}$ & 144 & 11.80 & $100,133, \mathbf{1 4 4}, 218$ & 2 & $10.732 \pm 0.785$ & $5.977 \pm 0.323 *$ & $5.387 \pm 0.257^{\#}$ & $7.584 \pm 0.239^{+}$ & $7.687 \pm 3.507$ \\
\hline 14 & 1-Monopalmitin & 371 & 45.26 & $103,129,205,371$ & 2 & $4.647 \pm 0.403$ & $3.781 \pm 1.278$ & $3.705 \pm 0.421^{\#}$ & $2.835 \pm 0.433^{+}$ & $4.372 \pm 1.145$ \\
\hline 15 & Linoleic acid & 75 & 37.82 & $67,75,81,337$ & 1 & $0.528 \pm 0.074$ & $0.394 \pm 0.089 *$ & $0.268 \pm 0.059$ \# & $0.357 \pm 0.044^{+}$ & $0.337 \pm 0.044$ \\
\hline 16 & Palmitic acid & 117 & 33.33 & $\mathbf{1 1 7}, 132,145,313$ & 1 & $0.673 \pm 0.049$ & $0.636 \pm 0.057$ & $0.649 \pm 0.053$ & $0.617 \pm 0.090$ & $0.707 \pm 0.080$ \\
\hline 17 & $\begin{array}{l}\text { Stearic acid } \\
\text { Organic acids }\end{array}$ & 117 & 38.66 & $117,132,145,341$ & 1 & $0.240 \pm 0.039$ & $0.367 \pm 0.083 *$ & $0.286 \pm 0.103$ & $0.267 \pm 0.081$ & $0.323 \pm 0.076$ \\
\hline 18 & Acetic acid & 177 & 8.12 & $133,161,177,205$ & 2 & $0.143 \pm 0.012$ & $0.124 \pm 0.008 *$ & $0.137 \pm 0.020$ & $0.143 \pm 0.016$ & $0.150 \pm 0.017$ \\
\hline 19 & Citric acid & 273 & 26.52 & $273,347,363,375$ & 4 & $120.814 \pm 4.491$ & $34.142 \pm 1.741 *$ & $50.563 \pm 1.841$ \# & $9.052 \pm 4.326^{+}$ & $114.971 \pm 4.669$ \\
\hline 20 & Fumaric acid & 245 & 15.46 & $115,132,143,245$ & 2 & $1.181 \pm 0.074$ & $0.043 \pm 0.005 *$ & $0.045 \pm 0.004$ & $0.287 \pm 0.033^{+}$ & $0.189 \pm 0.036$ \\
\hline 21 & Lactic acid & 117 & 7.73 & $117,133,191,219$ & 2 & $21.595 \pm 1.210$ & $602.047 \pm 19.653 *$ & $563.702 \pm 33.204$ \# & $547.899 \pm 14.857^{+}$ & $420.494 \pm 27.212$ \\
\hline 22 & Malic acid & 233 & 18.93 & $133,189,233,245$ & 3 & $89.641 \pm 5.199$ & ND & ND & $41.678 \pm 2.598^{+}$ & ND \\
\hline 23 & Malonic acid & 75 & 11.51 & $66,75,133,233$ & 2 & $0.441 \pm 0.035$ & $0.429 \pm 0.023$ & $0.425 \pm 0.028$ & $0.419 \pm 0.029$ & $0.533 \pm 0.115$ \\
\hline 24 & Succinic acid & 247 & 14.48 & $75,129,172,247$ & 2 & $1.544 \pm 0.071$ & $1.768 \pm 0.071 *$ & $2.462 \pm 0.108^{\#}$ & $56.616 \pm 3.446^{+}$ & $3.044 \pm 0.131^{\wedge}$ \\
\hline 25 & $\begin{array}{l}\text { Tartaric acid } \\
\text { Sugars }\end{array}$ & 292 & 22.43 & $189,219,292,423$ & 4 & $0.776 \pm 0.028$ & $0.602 \pm 0.084$ * & $0.638 \pm 0.037^{\#}$ & $0.686 \pm 0.019^{+}$ & $0.683 \pm 0.034$ \\
\hline 26 & Fructose & $\begin{array}{l}217 \\
103\end{array}$ & $\begin{array}{l}26.34 \\
28.01 \\
28.32\end{array}$ & $\begin{array}{l}204,217,319,437 \\
103,133,217,307\end{array}$ & $\begin{array}{c}5 \\
5(\mathrm{MeOX})\end{array}$ & $526.553 \pm 32.602$ & $553.891 \pm 8.751$ & $520.415 \pm 26.583$ & $512.824 \pm 9.722$ & $521.351 \pm 26.811$ \\
\hline 27 & Galactose & 204 & 28.89 & $129,191, \mathbf{2 0 4}, 217$ & 5 & $6.029 \pm 0.373$ & $5.561 \pm 0.336$ & $6.183 \pm 0.352$ & $4.300 \pm 0.139^{+}$ & $5.980 \pm 0.365$ \\
\hline 28 & Glucose & 204 & $\begin{array}{l}28.61 \\
31.40\end{array}$ & $129,191,204,217$ & 5 & $2905.897 \pm 138.257$ & $1486.807 \pm 39.829 *$ & $2156.601 \pm 58.234$ \# & $805.171 \pm 102.179^{+}$ & $2385.734 \pm 64.071$ \\
\hline & & 319 & 28.72 & $160,205,217,319$ & $5(\mathrm{MeOX})$ & & & & & \\
\hline 29 & Glucose-6-phosphate & 204 & $\begin{array}{l}40.26 \\
41.74\end{array}$ & $204,217,299,387$ & 6 & $0.329 \pm 0.032$ & $0.288 \pm 0.025 *$ & $0.316 \pm 0.021$ & $0.251 \pm 0.026^{+}$ & $0.618 \pm 0.058$ \\
\hline 30 & Sedoheptulose & 319 & $\begin{array}{l}35.74 \\
35.89\end{array}$ & $205,217,262,319$ & 6(MeOX) & $50.748 \pm 3.714$ & $42.976 \pm 1.234$ * & $47.967 \pm 1.442$ & $49.610 \pm 0.918$ & $44.614 \pm 3.511^{\wedge}$ \\
\hline
\end{tabular}


Table 1. Cont.

\begin{tabular}{|c|c|c|c|c|c|c|c|c|c|c|}
\hline No. & Compound & $m / z$ & $\begin{array}{c}\mathrm{RT} \\
(\mathrm{min})\end{array}$ & $\begin{array}{l}\text { Fragmentation } \\
\text { Ions }(\mathrm{m} / \mathrm{z})\end{array}$ & TMS & Control & $\begin{array}{l}\text { L. plantarum } \\
\text { (HY7712) }\end{array}$ & $\begin{array}{l}\text { L. plantarum } \\
\text { (HY7715) }\end{array}$ & $\begin{array}{l}\text { L. helveticus } \\
\text { (HY7801) }\end{array}$ & B. lactis (HY8002) \\
\hline 31 & Sucrose & 361 & 46.06 & $103,217, \mathbf{3 6 1}, 437$ & 8 & $437.362 \pm 192.453$ & $556.267 \pm 20.170$ & $535.635 \pm 35.941$ & $509.509 \pm 14.353$ & $507.215 \pm 24.494$ \\
\hline 32 & $\begin{array}{c}\text { Xylose } \\
\text { Sugar acids }\end{array}$ & 103 & 22.85 & $103,189,217,307$ & 4(MeOX) & $0.700 \pm 0.042$ & $0.686 \pm 0.104$ & $0.708 \pm 0.051$ & $0.715 \pm 0.047$ & $0.783 \pm 0.022$ \\
\hline 33 & Glyceric acid & 189 & 14.88 & $103, \mathbf{1 8 9}, 205,292$ & 3 & $0.268 \pm 0.024$ & ND & ND & ND & $0.148 \pm 0.016^{\wedge}$ \\
\hline 34 & $\begin{array}{l}\text { Threonic acid } \\
\text { Sugar alcohols }\end{array}$ & 292 & 20.66 & $117,205,220,292$ & 4 & $0.569 \pm 0.030$ & $0.455 \pm 0.031 *$ & $0.502 \pm 0.026^{\#}$ & $0.494 \pm 0.026^{+}$ & $0.554 \pm 0.029$ \\
\hline 35 & Erythritol & 217 & $\begin{array}{l}19.22 \\
19.39\end{array}$ & 103, 117, 205, 217 & 4 & $13.869 \pm 0.680$ & $1.057 \pm 0.045^{*}$ & $1.119 \pm 0.054$ \# & $6.792 \pm 0.327^{+}$ & $1.647 \pm 0.436$ \\
\hline 36 & Glycerol & 205 & 13.41 & $103,117,133,205$ & 3 & $16.011 \pm 0.751$ & $18.614 \pm 0.157 *$ & $18.262 \pm 0.573^{\#}$ & $16.246 \pm 0.480$ & $20.278 \pm 0.708^{\wedge}$ \\
\hline 37 & Mannitol & 319 & 29.66 & $103,205,217,319$ & 6 & $11.188 \pm 0.638$ & $9.783 \pm 0.434 *$ & $10.961 \pm 0.551$ & $10.554 \pm 0.242^{+}$ & $18.955 \pm 12.144$ \\
\hline 38 & Myo-Inositol & 305 & 34.45 & $191,217, \mathbf{3 0 5}, 318$ & 6 & $50.657 \pm 1.580$ & $45.353 \pm 1.026^{*}$ & $48.372 \pm 2.080^{\#}$ & $50.395 \pm 1.749$ & $49.615 \pm 1.095$ \\
\hline 39 & $\begin{array}{l}\text { Xylitol } \\
\text { Others }\end{array}$ & 217 & 24.25 & $103,205,217,307$ & 5 & $1.149 \pm 0.043$ & $1.050 \pm 0.050 *$ & $1.122 \pm 0.047$ & $1.096 \pm 0.051$ & $1.185 \pm 0.082$ \\
\hline 40 & Phosphoric acid & 299 & 13.30 & $133,211,299,314$ & 3 & $262.434 \pm 9.607$ & $186.249 \pm 12.682 *$ & $201.558 \pm 3.812^{\#}$ & $184.653 \pm 4.847^{+}$ & $217.457 \pm 7.081$ \\
\hline 41 & Uracil & 241 & 15.03 & $99,113, \mathbf{2 4 1}, 255$ & 2 & $0.075 \pm 0.011$ & $0.057 \pm 0.005^{*}$ & $0.054 \pm 0.006^{\#}$ & $0.835 \pm 0.046^{+}$ & $0.099 \pm 0.064$ \\
\hline
\end{tabular}

(IL. plantarum (HY7712), *; L. plantarum (HY7715), \#; spectrum); TMS, trimethylsilylation; MeOX, methoxylamine hydrochloride. 
Table 2. Relative levels of lipids in the fermented and non-fermented VJs, as detected by DI-MS.

\begin{tabular}{|c|c|c|c|c|c|c|c|c|}
\hline No. & Lipid Species & Ion Species & $m / z$ & Control & $\begin{array}{l}\text { L. plantarum } \\
\text { (HY7712) }\end{array}$ & $\begin{array}{l}\text { L. plantarum } \\
\text { (HY7715) }\end{array}$ & $\begin{array}{l}\text { L. helveticus } \\
\text { (HY7801) }\end{array}$ & $\begin{array}{l}\text { B. lactis } \\
\text { (HY8002) }\end{array}$ \\
\hline & \multicolumn{8}{|c|}{$\begin{array}{l}\text { Positive ion mode } \\
\end{array}$} \\
\hline & \multicolumn{8}{|c|}{ Monogalactosyldiacylglycerol (MGDG) } \\
\hline 1 & MGDG 18:2/18:3 & {$[\mathrm{M}+\mathrm{Na}]^{+}$} & 799 & $1.93 \pm 0.62$ & $1.74 \pm 0.43$ & $1.67 \pm 0.47$ & $1.88 \pm 0.31$ & $1.55 \pm 0.52$ \\
\hline 2 & MGDG 18:2/18:2 & {$[\mathrm{M}+\mathrm{Na}]^{+}$} & 801 & $14.42 \pm 4.00$ & $13.24 \pm 3.19$ & $12.68 \pm 3.14$ & $13.64 \pm 1.71$ & $11.89 \pm 3.22$ \\
\hline \multirow[t]{2}{*}{3} & MGDG 18:1/18:2 & {$[\mathrm{M}+\mathrm{Na}]^{+}$} & 803 & $11.51 \pm 3.39$ & $10.38 \pm 1.90$ & $9.52 \pm 1.93$ & $10.37 \pm 0.93$ & $9.28 \pm 2.10$ \\
\hline & \multicolumn{8}{|c|}{ Lysophosphatidylcholine (Lyso-PC) } \\
\hline 4 & Lyso-PC 18:2 & {$[\mathrm{M}+\mathrm{H}]^{+}$} & 520 & $5.30 \pm 1.04$ & $4.18 \pm 1.05$ & $4.36 \pm 0.96$ & $4.56 \pm 0.70$ & $3.73 \pm 0.70^{\wedge}$ \\
\hline 5 & Lyso-PC 18:1 & {$[\mathrm{M}+\mathrm{H}]^{+}$} & 522 & $6.56 \pm 1.60$ & $6.53 \pm 1.16$ & $6.87 \pm 1.16$ & $6.38 \pm 0.64$ & $6.37 \pm 1.01$ \\
\hline \multirow{2}{*}{6} & Lyso-PC 22:5 & {$[\mathrm{M}+\mathrm{Na}]^{+}$} & 592 & $2.81 \pm 0.61$ & $2.55 \pm 0.52$ & $2.31 \pm 0.52$ & $2.80 \pm 0.38$ & $2.39 \pm 0.56$ \\
\hline & \multicolumn{8}{|c|}{ Phosphatidylcholine (PC) } \\
\hline \multirow[t]{2}{*}{7} & PC 18:2/18:2 & {$[\mathrm{M}+\mathrm{H}]^{+}$} & 782 & $4.29 \pm 0.50$ & $4.16 \pm 0.23$ & $4.08 \pm 0.28$ & $4.06 \pm 0.30$ & $4.10 \pm 0.30$ \\
\hline & \multicolumn{8}{|c|}{ Phosphatidylethanolamine (PE) } \\
\hline 8 & PE 16:0/20:0 & {$[\mathrm{M}+\mathrm{H}]^{+}$} & 748 & $1.17 \pm 0.30$ & $1.05 \pm 0.11$ & $0.98 \pm 0.14$ & $1.08 \pm 0.11$ & $1.08 \pm 0.22$ \\
\hline & \multicolumn{8}{|c|}{ Triacylglycerol (TG) } \\
\hline 9 & TG 16:0/18:2/18:2 & {$\left[\mathrm{M}+\mathrm{NH}_{4}\right]^{+}$} & 872 & $3.88 \pm 0.26$ & $4.19 \pm 0.42$ & $4.13 \pm 0.49$ & $4.17 \pm 0.59$ & $4.09 \pm 0.31$ \\
\hline 10 & TG $18: 2 / 18: 2 / 18: 3$ & {$\left[\mathrm{M}+\mathrm{NH}_{4}\right]^{+}$} & 894 & $2.85 \pm 0.19$ & $3.03 \pm 0.32$ & $3.01 \pm 0.30$ & $3.03 \pm 0.39$ & $2.98 \pm 0.28$ \\
\hline 11 & TG 18:2/18:2/18:2 & {$\left[\mathrm{M}+\mathrm{NH}_{4}\right]^{+}$} & 896 & $9.76 \pm 0.61$ & $10.66 \pm 1.03$ & $10.45 \pm 1.07$ & $10.49 \pm 1.31$ & $10.47 \pm 0.83$ \\
\hline 12 & TG 18:1/18:2/18:2 & {$\left[\mathrm{M}+\mathrm{NH}_{4}\right]^{+}$} & 898 & $3.33 \pm 0.18$ & $3.73 \pm 0.35$ & $3.68 \pm 0.46$ & $3.64 \pm 0.56$ & $3.64 \pm 0.28$ \\
\hline \multirow[t]{3}{*}{13} & TG $18: 1 / 18: 1 / 18: 2$ & {$\left[\mathrm{M}+\mathrm{NH}_{4}\right]^{+}$} & 900 & $1.30 \pm 0.04$ & $1.46 \pm 0.16$ & $1.46 \pm 0.21$ & $1.41 \pm 0.20$ & $1.42 \pm 0.13$ \\
\hline & \multicolumn{8}{|c|}{ Negative ion mode } \\
\hline & \multicolumn{8}{|c|}{ Phosphatic acid (PA) } \\
\hline 14 & PA 16:0/18:2 & {$[\mathrm{M}-\mathrm{H}]^{-}$} & 671 & $1.81 \pm 0.27$ & $1.76 \pm 0.21$ & $1.81 \pm 0.43$ & $1.84 \pm 0.30$ & $1.67 \pm 0.13$ \\
\hline \multirow[t]{2}{*}{15} & PA 18:2/18:2 & {$[\mathrm{M}-\mathrm{H}]^{-}$} & 695 & $1.74 \pm 0.27$ & $1.56 \pm 0.35$ & $1.48 \pm 0.35$ & $1.69 \pm 0.32$ & $1.44 \pm 0.19$ \\
\hline & \multicolumn{8}{|c|}{ Phosphatidylethanolamine (PE) } \\
\hline 16 & PE 16:0/18:2 & {$[\mathrm{M}-\mathrm{H}]^{-}$} & 714 & $1.46 \pm 0.06$ & $1.35 \pm 0.08$ & $1.35 \pm 0.04^{\#}$ & $1.41 \pm 0.09$ & $1.41 \pm 0.10$ \\
\hline 17 & PE 18:2/18:2 & {$[\mathrm{M}-\mathrm{H}]^{-}$} & 738 & $1.23 \pm 0.05$ & $3.75 \pm 2.19^{*}$ & $2.72 \pm 0.69^{\#}$ & $4.43 \pm 2.06^{+}$ & $1.96 \pm 0.31$ \\
\hline 18 & PE 18:1/18:2 & {$[\mathrm{M}-\mathrm{H}]^{-}$} & 740 & $0.73 \pm 0.05$ & $1.18 \pm 0.83$ & $0.76 \pm 0.17$ & $1.15 \pm 0.70^{+}$ & $0.62 \pm 0.09^{\wedge}$ \\
\hline \multirow[t]{2}{*}{19} & PE 18:0/18:2 & {$[\mathrm{M}-\mathrm{H}]^{-}$} & 742 & $4.50 \pm 0.84$ & $1.51 \pm 0.85 *$ & $1.22 \pm 0.49^{\#}$ & $1.01 \pm 0.51^{+}$ & $2.29 \pm 0.75$ \\
\hline & \multicolumn{8}{|c|}{ Phosphatidylglycerol (PG) } \\
\hline 20 & PG 16:0/18:2 & \multicolumn{6}{|c|}{ Phosphatidylserine (PS) } & $1.47 \pm 0.20$ \\
\hline 21 & PS 18:2/20:0 & {$[\mathrm{M}-\mathrm{H}]^{-}$} & 814 & $0.23 \pm 0.04$ & $0.33 \pm 0.21$ & $0.21 \pm 0.05$ & $0.15 \pm 0.08^{+}$ & $0.27 \pm 0.04$ \\
\hline 22 & PS $18: 2 / 22: 0$ & {$[\mathrm{M}-\mathrm{H}]^{-}$} & 842 & $0.75 \pm 0.12$ & $0.98 \pm 0.18$ * & $0.92 \pm 0.09^{\#}$ & $0.91 \pm 0.11$ & $0.98 \pm 0.14$ \\
\hline & \multicolumn{8}{|c|}{ Phosphatidylinositol (PI) } \\
\hline 23 & PI 16:0/18:2 & {$[\mathrm{M}-\mathrm{H}]^{-}$} & 833 & $6.34 \pm 0.86$ & $7.59 \pm 1.00$ & $7.44 \pm 1.13$ & $8.04 \pm 1.21^{+}$ & $7.50 \pm 1.05$ \\
\hline 24 & PI 16:0/18:1 & {$[\mathrm{M}-\mathrm{H}]^{-}$} & 835 & $1.01 \pm 0.15$ & $1.32 \pm 0.21 *$ & $1.34 \pm 0.23^{\#}$ & $3.58 \pm 0.21^{+}$ & $1.24 \pm 0.15{ }^{\wedge}$ \\
\hline
\end{tabular}

Mann-Whitney test was performed to detect significant differences between fermented VJs and the control. (\{L. plantarum (HY7712), *; L. plantarum (HY7715), \#; L. helveticus (HY7801), +; B. lactis (HY8002), ^\}, $p<0.05)$. 
Table 3. Relative levels of carotenoids in the fermented and non-fermented VJs, as detected by LC-MS.

\begin{tabular}{ccccccccc}
\hline Compound & Formula & $\begin{array}{c}\text { RT } \\
(\mathbf{m i n})\end{array}$ & $\begin{array}{c}\mathrm{m} / \boldsymbol{z} \\
{[\mathbf{M}+\mathbf{H}]}\end{array}$ & Control & $\begin{array}{c}\text { L. plantarum } \\
\text { (HY7712) }\end{array}$ & $\begin{array}{c}\text { L. plantarum } \\
\text { (HY7715) }\end{array}$ & $\begin{array}{c}\text { L. helveticus } \\
\text { (HY7801) }\end{array}$ & $\begin{array}{c}\text { B. lactis } \\
\text { (HY8002) }\end{array}$ \\
\hline LUT & $\mathrm{C}_{40} \mathrm{H}_{56} \mathrm{O}_{2}$ & 5.61 & 569.4 & $4.0 \pm 0.2$ & $4.2 \pm 0.3$ & $4.2 \pm 0.1$ & $2.6 \pm 0.5^{+}$ & $4.4 \pm 0.5$ \\
LYC & $\mathrm{C}_{40} \mathrm{H}_{56}$ & 9.32 & 537.4 & $30.8 \pm 0.9$ & $36.3 \pm 0.6^{*}$ & $45.1 \pm 3.0^{\#}$ & $34.0 \pm 9.7$ & $38.2 \pm 2.1^{*}$ \\
$\alpha-$ CAR & $\mathrm{C}_{40} \mathrm{H}_{56}$ & 10.95 & 537.4 & $50.9 \pm 3.5$ & $60.9 \pm 5.7^{*}$ & $69.0 \pm 5.9^{\#}$ & $61.8 \pm 6.2^{+}$ & $54.8 \pm 4.0$ \\
$\beta-$ CAR & $\mathrm{C}_{40} \mathrm{H}_{56}$ & 11.12 & 537.4 & $113.1 \pm 7.6$ & $134.4 \pm 13.4^{*}$ & $147.7 \pm 13.5^{\#}$ & $111.3 \pm 12.6$ & $120.2 \pm 8.7$ \\
\hline
\end{tabular}

Mann-Whitney test was performed to detect significant differences between fermented VJs and the control

(\{L. plantarum (HY7712), *; L. plantarum (HY7715), \#; L. helveticus (HY7801), +; B. lactis (HY8002), \} , $p<0.05)$. LUT,

lutein; LYC, lycopene; $\alpha$-CAR, $\alpha$-carotene; $\beta$-CAR, $\beta$-carotene; RT, retention time.

\subsection{Probiotic Fermentation of VJ Alters its Metabolic and Lipidomic Profiles}

The metabolic and lipidomic data for the different juices were clearly distinguished in the PCA and PLS-DA score plots Figure 1. The metabolic and lipidomic profiles of VJs fermented by the two L. plantarum strains (HY7712 and HY7715) were similar and clearly distinguishable from those of VJ fermented by L. helveticus HY7801. Moreover, the data for B. lactis HY8002-fermented juice differed from those for Lactobacillus HY7712, HY7715, and HY7801-fermented juices.

Our findings confirm that the metabolites and lipids present in fermented VJ differ depending on which probiotic strain was used to produce it. It follows that different probiotics might utilize different nutritional compounds of the juice during fermentation. A metabolic pathway analysis of the 41 metabolites identified by GC-MS showed that the following processes were activated after probiotic fermentation of VJ: alanine, aspartate, and glutamate metabolism; glycine, serine, and threonine metabolism; the citrate cycle; aminoacyl-tRNA biosynthesis; starch and sucrose metabolism; and arginine and proline metabolism (Table 4). The main metabolites of these pathways were largely consistent with the 27 metabolites with a VIP (Variable Importance in the Projection) score above 1.0 in the PLS-DA model (Table 5). The relative abundance of the altered metabolites and lipids and the related metabolic pathways are presented in Figure 2.
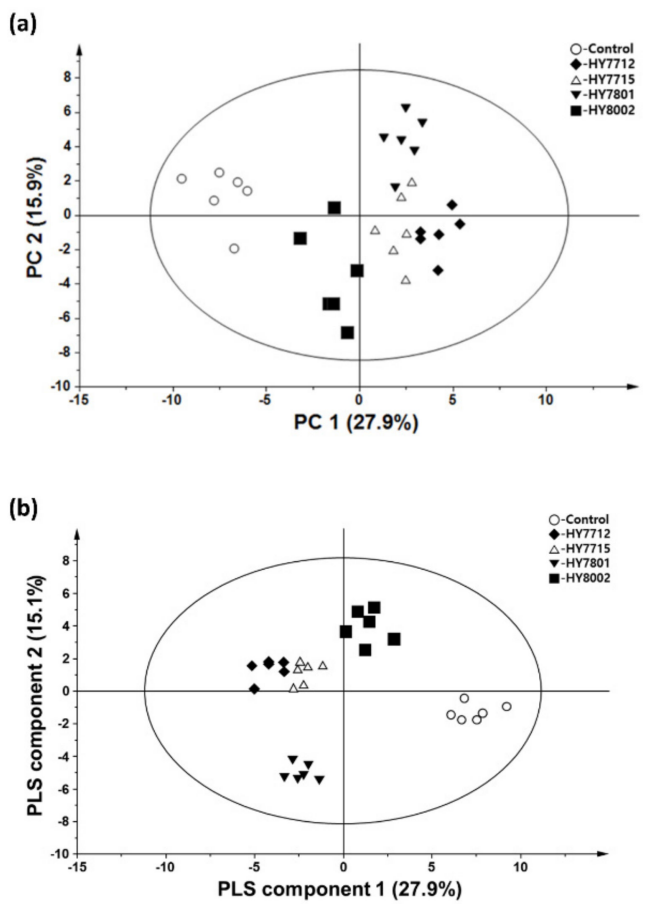

Figure 1. Metabolic and lipidomic data for the fermented and non-fermented VJs. (a) PCA score plot. (b) Partial least squares-discriminant analysis (PLS-DA) score plot. $n=6$ in each group. 
Table 4. Main metabolic pathways activated in the fermented and non-fermented VJs.

\begin{tabular}{|c|c|c|c|c|c|c|}
\hline No. & Pathway Name & Compound $^{a}$ & Total $^{b}$ & Hits $^{c}$ & $p^{d}$ & Impact $^{e}$ \\
\hline 1 & $\begin{array}{l}\text { Alanine, aspartate } \\
\text { and glutamate } \\
\text { metabolism }\end{array}$ & $\begin{array}{l}\text { alanine, aspartic acid, glutamic acid, } \\
\text { asparagine, succinic acid, } \\
\gamma \text {-aminobutanoic acid, fumaric acid }\end{array}$ & 20 & 7 & $8.18 \times 10^{-6}$ & 0.60 \\
\hline 2 & $\begin{array}{l}\text { Glycine, serine and } \\
\text { threonine } \\
\text { metabolism }\end{array}$ & $\begin{array}{c}\text { glycine, serine, threonine, glyceric acid, } \\
\text { aspartic acid }\end{array}$ & 28 & 5 & $5.40 \times 10^{-3}$ & 0.42 \\
\hline 3 & $\begin{array}{c}\text { Citrate cycle (TCA } \\
\text { cycle) }\end{array}$ & $\begin{array}{l}\text { citric acid, fumaric acid, malic acid, } \\
\text { succinic acid }\end{array}$ & 20 & 4 & $8.64 \times 10^{-3}$ & 0.20 \\
\hline 4 & $\begin{array}{l}\text { Aminoacyl-tRNA } \\
\text { biosynthesis }\end{array}$ & $\begin{array}{l}\text { asparagine, glycine, aspartic acid, } \\
\text { serine, valine, alanine, threonine, } \\
\text { proline, glutamic acid, isoleucine }\end{array}$ & 66 & 10 & $2.52 \times 10^{-4}$ & 0.18 \\
\hline 5 & $\begin{array}{l}\text { Starch and sucrose } \\
\text { metabolism }\end{array}$ & $\begin{array}{l}\text { fructose, glucose, glucose-6-phosphate, } \\
\text { sucrose, xylose }\end{array}$ & 30 & 5 & $7.35 \times 10^{-3}$ & 0.15 \\
\hline 6 & $\begin{array}{l}\text { Arginine and } \\
\text { proline metabolism }\end{array}$ & $\begin{array}{l}\text { aspartic acid, fumaric acid, proline, } \\
\text { glutamic acid, } \gamma \text {-aminobutanoic acid }\end{array}$ & 40 & 5 & $2.47 \times 10^{-2}$ & 0.10 \\
\hline
\end{tabular}

${ }^{a}$ The names of matched compounds from the fermented and non-fermented VJs. ${ }^{b}$ Total number of compounds in the pathway. ${ }^{c}$ Number of matched compounds. ${ }^{d}$ Original $p$ value calculated from the uploaded data. ${ }^{e}$ Pathway impact value calculated from pathway topology analysis.

Table 5. Metabolites and lipids with VIP values $>1.0$ in the PLS-DA model.

\begin{tabular}{ccc}
\hline No. & Compound & VIP Value \\
\hline 1 & $\gamma$-aminobutanoic acid & 1.71 \\
2 & Glycine & 1.51 \\
3 & Glucose-6-phosphate & 1.43 \\
4 & Uracil & 1.42 \\
5 & $\beta$-alanine & 1.42 \\
6 & Succinic acid & 1.41 \\
7 & Linoleic acid & 1.41 \\
8 & Aspartic acid & 1.39 \\
9 & Galactose & 1.38 \\
10 & Proline & 1.36 \\
11 & Asparagine & 1.35 \\
12 & Serine & 1.33 \\
13 & Glycerol & 1.29 \\
14 & Sedoheptulose & 1.28 \\
15 & Glucose & 1.23 \\
16 & Myo-inositol & 1.23 \\
17 & Malic acid & 1.20 \\
18 & Erythritol & 1.16 \\
19 & Isoleucine & 1.14 \\
20 & Threonine & 1.13 \\
21 & Fructose & 1.13 \\
22 & Fumaric acid & 1.12 \\
23 & Citric acid & 1.08 \\
24 & Glyceric acid & 1.08 \\
25 & Malonic acid & 1.03 \\
26 & Xylitol & 1.03 \\
27 & Glutamic acid & 1.01 \\
28 & Phosphatidylethanolamine (PE) 18:0/18:2 & 1.00 \\
29 & & 1.00 \\
\hline & &
\end{tabular}




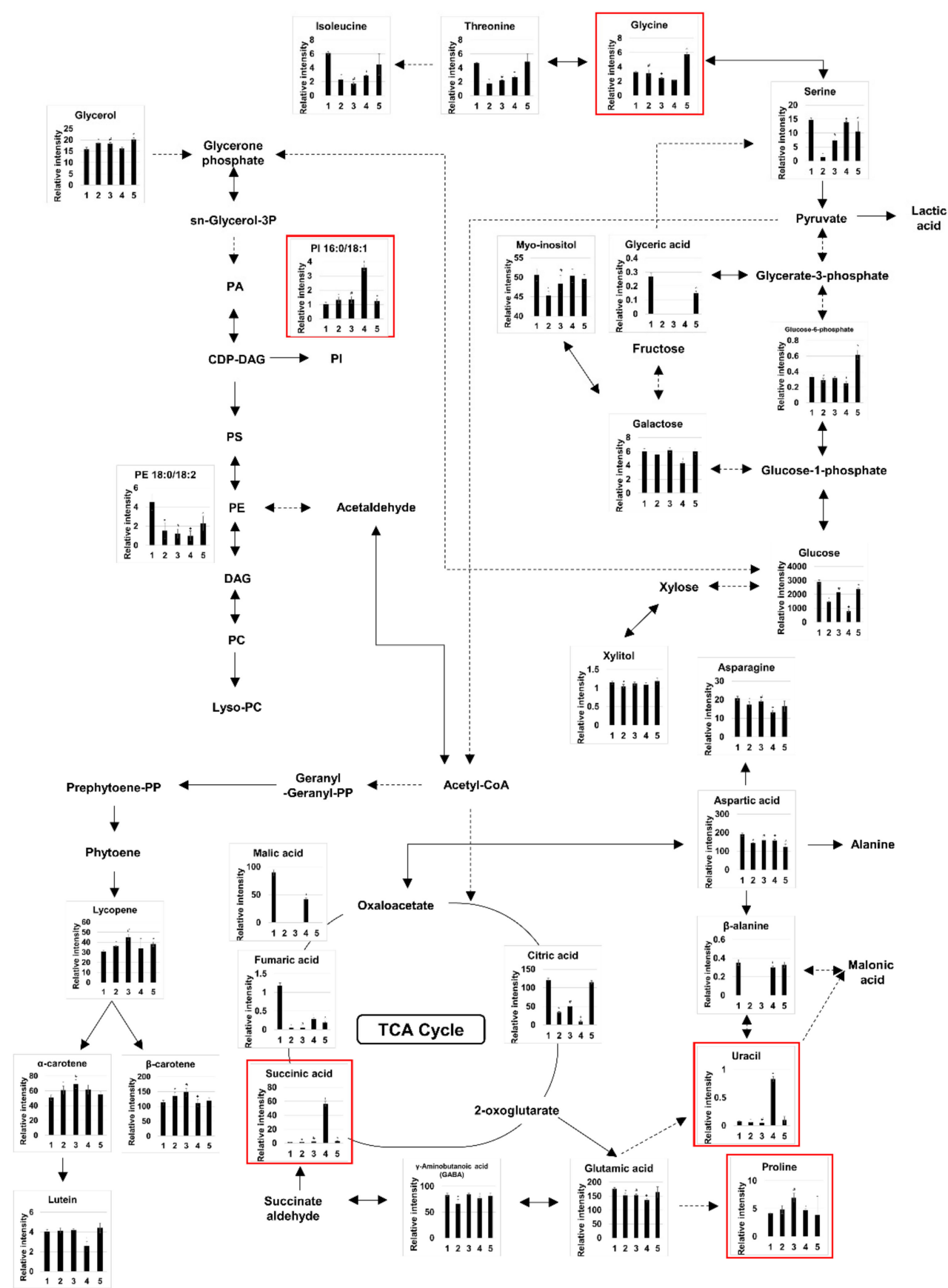

Figure 2. Relative intensity of metabolites, lipids, and carotenoids in the fermented and non-fermented VJs and the related metabolic pathways. Citrate cycle (TCA cycle) and the alanine, aspartate, and glutamate metabolism pathways. Glycine, serine, and threonine metabolism and the glycolysis pathways. Glycerophospholipid metabolism pathway. Carotenoid biosynthetic pathway. Significant differences $(p<0.05)$ between fermented VJs and the control (1) are indicated as follows: (\{(2) L. plantarum (HY7712), *; (3) L. plantarum (HY7715), \#; (4) L. helveticus (HY7801), +; (5) B. lactis (HY8002), ^\}, p < 0.05). PC, phosphatidylcholine; PE, phosphatidylethanolamine; PI, phosphatidylinositol; PS, phosphatidylserine; PA, phosphatidic acid; DAG, diacylglycerol; CDP-DAG, cytidine diphosphate-diacylglycerol. The major differential compounds (4 metabolites and 1 intact lipid) between the fermented and non-fermented VJs are shown in red. 


\section{Discussion}

We hypothesized that the change in the levels of metabolites, lipids, and carotenoids in VJ might be affected by the activities of probiotic enzymes. Galactosidases, glucosidase, lipase, and leucine aminopeptidase are present in human Bifidobacteria [28], while $\beta$-galactosidase and $\beta$-d-phosphogalactoside galactohydrolase were detected in Lactobacillus strains [29]. Malolactic enzymes purified from L. plantarum convert l-malate to l-lactate [30]. In addition, the production of exopolysaccharide by L. rhamnosus and L. sakei is associated with a number of enzymes, including $\alpha$-d-glucosidase, $\beta$-d-glucuronidase, and $\alpha$-phosphoglucomutase [31,32].

Our results suggest that the production of certain metabolites is markedly enhanced because of the activation of multiple metabolic pathways (Figure 2). In HY7801-fermented VJ, the level of uracil was higher by 11.1 times than in the control. Uracil is a pyrimidine nucleobase that binds to adenine in RNA [33] and is essential for the growth of lactobacilli [34]. Orostachys japonicus A. Berger, commonly called rock pine, fermented with L. plantarum displays 4.73 times the uracil content of non-fermented Orostachys japonicus A. Berger [35], and it is thought that uracil affects the growth and antibacterial activity of this probiotic strain [36]. Uracil and glycerol in soymilk fermented with L. plantarum show antihypertensive effects [37]. Moreover, uracil could play an important role in the detoxification of carcinogens such as tobacco smoke [38]. Kim et al. [39] suggested that the anti-inflammatory effects of uracil in garlic might be mediated by modulating NF- $\mathrm{KB}$ signaling, and Zimin et al. [40] revealed that acid derivatives of uracil might also exhibit anti-inflammatory properties. We surmised that the anti-inflammatory effects of uracil could be related to the findings of previous studies on L. helveticus HY7801. Research in animal models has shown L. helveticus HY7801 to have a number of anti-inflammatory effects. Orally administered HY7801 regulates immune biomarkers, including TNF- $\alpha$, IFN- $\gamma$, IL-17A, IL-10, and IL-12, and might be useful in the treatment of rheumatoid arthritis [41]. In addition, oral administration of HY7801 improves vulvovaginal candidiasis by inhibiting the survival of Candida albicans and down-regulating TNF- $\alpha$, COX-2, iNOS, and IL-1 $\beta$ levels [42]. According to Hong et al. [43], administration of HY7801 to mice with colitis altered their intestinal microbiota and fecal metabolite levels.

In HY7801-fermented VJ, the level of succinic acid was also higher by 36.67 times than in the control. Succinic acid is an intermediate of the tricarboxylic acid (TCA) cycle and the end-product of anaerobic fermentation [44] and is widely used in the agricultural, food, and pharmaceutical industries [45]. L. helveticus produces succinic acids through citric acid metabolism, which are thought to contribute to the flavor profiles of Emmental and cheddar cheese [46]. In addition, Streptococcus lactis (recently Lactococcus lactis) converts fumaric acid to succinic acid [47]. Succinic acid production by lactic acid bacteria could be a response to stalled growth resulting from nutrient depletion [48]. Therefore, it is possible that decreasing nutrient levels during VJ fermentation halted the growth of HY7801, thereby stimulating the production of succinic acid. Succinic acid has numerous beneficial effects on human health. Consumption of succinic acid improves nerve cell function [49] and is therapeutic in patients with brain damage [50]. Furthermore, succinic acid is a proposed treatment for cervical cancer [51] and might have antioxidant capabilities [52].

In addition, in HY7801-fermented VJ, the level of PI 16:0/18:1 was higher by 3.54 times than in the control. PI is produced from CDP-diacylglycerol and plays important roles in cell signaling, cell wall structure, and protein metabolism [53,54]. Increased PI production by Saccharomyces cerevisiae might be a response to nutrient exhaustion and entry into the stationary phase [53]. Ethanol production by Saccharomyces strains is affected by increased PI levels [55]. We speculate that the growth of HY7801 might have been halted because of the depletion of nutrients required for fermentation. Dietary PI supplementation shows promise in the treatment of various diseases, such as diabetic neuropathy [56]. Küllenberg et al. [57] suggested that consumption of phospholipids could have a positive effect on inflammation, cancer, cardiovascular diseases, and liver disease. Notably, oral administration of PI increases the level of high-density lipoprotein-cholesterol (HDL-C) in human plasma [58]. 
Moreover, proline content of HY7715-fermented VJ was 1.66 times higher than that of non-fermented juice. Proline is an amino acid that participates in protein synthesis [59-61]. Its production is upregulated in plants in response to various stresses, including temperature and reactive oxygen species [62], and Saccharomyces cerevisiae might synthesize it to adapt to stress during fermentation [63]. Proline production could be related to changes in glutamic acid levels during Kurthia catenaforma fermentation [64]. Proline is an essential amino acid that participates in collagen synthesis [65] and is useful for intestinal health [66].

Glycine content of HY8002-fermented VJ was also 1.76 times higher than that of non-fermented juice. Glycine is essential for the formation of secondary protein structure [67]. Moreover, proliferating lactic acid bacteria increase the capacity for glycine production in an L. salivarius and P. acidilactici co-culture [68]. Glycine consumption reportedly reduces fatigue [69] and prevents skin cancer in animal models [70].

Carotenoids are a group of bioactive tetraterpenoids that exhibit antioxidant properties [71]. Among the carotenoids, lycopene has a role in the prevention of cancer and cardiovascular disease [72], while $\beta$-carotene is necessary for the maintenance of skin and mucous membranes, and visual adaptation to the dark. The latter is a functional ingredient approved by the Korean Ministry of Food and Drug Safety. Lycopene, $\alpha$-carotene, and $\beta$-carotene levels were significantly higher in L. plantarum HY7712 and HY7715-fermented juices than in non-fermented juice (Figure 2). Additionally, B. lactis HY8002 and L. helveticus HY7801-inoculated juices had increased levels of lycopene and $\alpha$-carotene, respectively. The latter juice also displayed a lower lutein content than the control. Tomato pulp fermented with Lactobacillus sakei, Pediococcus acidilactici, and Pediococcus pentosaceus has altered levels of lycopene and $\beta$-carotene [73]. L. plantarum produces the C30 carotenoid 4,4'-diaponeurosporene [74] and the triterpenoid carotenoid 4,4'-diaponeurosporene. On the other hand, Sanchez-Contreras et al. [75] suggested that lutein might be utilized as a carbon source for the growth of microorganisms. In this study, lutein might also be utilized for the growth of L. helveticus HY7801, and we thought that this metabolism in HY7801 could be related to the increase of succinic acid and PI under nutritional depletion in the fermented VJ $[48,53]$. Consistent with the findings of previous studies, probiotic fermentation of VJ modified its carotenoid content. We hypothesize that the antioxidative effects of elevated carotenoid levels in probiotic VJ are related to the immunity enhancement observed in a mouse model treated with L. plantarum HY7712. Orally administered HY7712 restores natural killer cells damaged by $\gamma$-irradiation [76] and accelerates the recovery of immunosuppression caused by the anticancer drug cyclophosphamide [77].

\section{Conclusions}

In this study, VJ was fermented with four probiotic strains, L. plantarum HY7712, L. plantarum HY7715, L. helveticus HY7801, and B. lactis HY8002, and the metabolite, lipid, and carotenoid content of each juice were analyzed by GC-MS, DI-MS, and LC-MS. The carotenoids, including lycopene, $\alpha$-carotene, and $\beta$-carotene levels, were higher in VJ fermented with L. plantarum strains (HY7712 and HY7715) than in the control. Particularly, the levels of uracil and succinic acid were increased in HY7801 fermented VJ, while Proline was also elevated in HY7715. In addition, glycine was increased in VJ fermented with HY8002. We also revealed that a number of metabolic pathways were activated in probiotics during the fermentation process, including the citrate cycle; alanine, aspartate, and glutamate metabolism; glycine, serine, and threonine metabolism; glycolysis; and carotenoid pathways. Compared to previous studies [14-18], we highlighted that three kinds of MS-platforms were applied to analyze many different kinds of metabolites, lipids, and carotenoids of probiotic-fermented VJs, and relative amounts of differentiated biological substances produced by three Lactobacillus strains and one Bifidobacterium strain were also investigated. Thus, we confirmed that metabolomics and lipidomics are the effective approach to provide more scientific evidence for discovering new beneficial effects of probiotics. Furthermore, we advanced the understanding of how fermented foods mediate their health benefits by revealing the metabolic changes that occur during probiotic fermentation. 
Author Contributions: Conceptualization, H.-J.C., S.-I.S., and H.-K.C.; methodology, H.-J.C., H.L., S.-I.S., and H.-K.C.; validation, H.-J.C. and H.L.; formal analysis, H.-J.C. and H.L.; investigation, H.-J.C. and H.L.; resources, H.-J.C., G.N., H.J., D.-G.K., and S.-E.J.; writing—original draft preparation, H.-J.C. and H.-K.C.; writing-review and editing, H.-J.C., H.L., and H.-K.C.; visualization, H.-J.C. and H.L.; supervision, H.-K.C.; project administration, H.-J.C., H.L., I.-d.C., S.-I.S., J.-H.L., J.-H.S., and H.-K.C. All authors have read and agreed to the published version of the manuscript.

Funding: This work was supported by the National Research Foundation of Korea (NRF) grant funded by the Korean government (MSIP) (NRF-2015R1A5A1008958).

Acknowledgments: This work was supported by the National Research Foundation of Korea (NRF) grant funded by the Korean government (MSIP) (NRF-2015R1A5A1008958).

Conflicts of Interest: The authors declare no conflict of interest.

\section{References}

1. FAO/WHO. Report of a joint FAO/WHO working group on drafting guidelines for the evaluation of probiotics in food. In Guidelines for the Evaluation of Probiotics in Food; FAO/WHO: London, ON, Canada, 2002.

2. Kechagia, M.; Basoulis, D.; Konstantopoulou, S.; Dimitriadi, D.; Gyftopoulou, K.; Skarmoutsou, N.; Fakiri, E.M. Health benefits of probiotics: A review. ISRN Nutr. 2013. [CrossRef]

3. Marco, M.L.; Heeney, D.; Binda, S.; Cifelli, C.J.; Cotter, P.D.; Foligne, B.; Ganzle, M.; Kort, R.; Pasin, G.; Pihlanto, A.; et al. Health benefits of fermented foods: Microbiota and beyond. Curr. Opin. Biotechnol. 2017, 44, 94-102. [CrossRef] [PubMed]

4. Khalesi, S.; Bellissimo, N.; Vandelanotte, C.; Williams, S.; Stanley, D.; Irwin, C. A review of probiotic supplementation in healthy adults: Helpful or hype? Eur. J. Clin. Nutr. 2019, 73, 24-37. [CrossRef] [PubMed]

5. Henning, S.M.; Yang, J.; Shao, P.; Lee, R.P.; Huang, J.; Ly, A.; Hsu, M.; Lu, Q.Y.; Thames, G.; Heber, D.; et al. Health benefit of vegetable/fruit juice-based diet: Role of microbiome. Sci. Rep. 2017, 7, 2167. [CrossRef] [PubMed]

6. Havas, P.; Kun, S.; Styevkó, G.; Slačanac, V.; Hardi, J.; Rezessy-Szabó, J. Fruit and vegetable juice fermentation with bifidobacteria. Acta Alimentaria 2014, 43, 64-72. [CrossRef]

7. Alissa, E.M.; Ferns, G.A. Dietary fruits and vegetables and cardiovascular diseases risk. Crit. Rev. Food Sci. Nutr. 2017, 57, 1950-1962. [CrossRef]

8. Kosewski, G.; Gorna, I.; Boleslawska, I.; Kowalowka, M.; Wieckowska, B.; Glowka, A.K.; Morawska, A.; Jakubowski, K.; Dobrzynska, M.; Miszczuk, P.; et al. Comparison of antioxidative properties of raw vegetables and thermally processed ones using the conventional and sous-vide methods. Food Chem. 2018, 240, 1092-1096. [CrossRef]

9. Imran, M.; Rauf, A.; Abu-Izneid, T.; Nadeem, M.; Shariati, M.A.; Khan, I.A.; Imran, A.; Orhan, I.E.; Rizwan, M.; Atif, M.; et al. Luteolin, a flavonoid, as an anticancer agent: A review. Biomed. Pharmacother. 2019, 112, 108612. [CrossRef]

10. Di Cagno, R.; Coda, R.; De Angelis, M.; Gobbetti, M. Exploitation of vegetables and fruits through lactic acid fermentation. Food Microbiol. 2013, 33, 1-10. [CrossRef]

11. Swain, M.R.; Anandharaj, M.; Ray, R.C.; Parveen Rani, R. Fermented fruits and vegetables of Asia: A potential source of probiotics. Biotechnol. Res. Int. 2014. [CrossRef]

12. Corona, O.; Randazzo, W.; Miceli, A.; Guarcello, R.; Francesca, N.; Erten, H.; Moschetti, G.; Settanni, L. Characterization of kefir-like beverages produced from vegetable juices. LWT Food Sci. Technol. 2016, 66, 572-581. [CrossRef]

13. Kim, S.Y. Production of fermented kale juices with Lactobacillus strains and nutritional composition. Prev. Nutr. Food Sci. 2017, 22, 231-236. [PubMed]

14. Tomita, S.; Saito, K.; Nakamura, T.; Sekiyama, Y.; Kikuchi, J. Rapid discrimination of strain-dependent fermentation characteristics among Lactobacillus strains by NMR-based metabolomics of fermented vegetable juice. PLoS ONE 2017, 12, e0182229. [CrossRef]

15. Yang, K.; Xu, M.; Zhong, F.; Zhu, J. Rapid differentiation of Lactobacillus species via metabolic profiling. J. Microbiol. Methods 2018, 154, 147-155. [CrossRef] [PubMed]

16. Filannino, P.; Cardinali, G.; Rizzello, C.G.; Buchin, S.; De Angelis, M.; Gobbetti, M.; Di Cagno, R. Metabolic responses of Lactobacillus plantarum strains during fermentation and storage of vegetable and fruit juices. Appl. Environ. Microbiol. 2014, 80, 2206-2215. [CrossRef] [PubMed] 
17. Tomita, S.; Nakamura, T.; Okada, S. NMR- and GC/MS-based metabolomic characterization of sunki, an unsalted fermented pickle of turnip leaves. Food Chem. 2018, 258, 25-34. [CrossRef] [PubMed]

18. Park, S.E.; Seo, S.H.; Lee, K.I.; Na, C.S.; Son, H.S. Metabolite profiling of fermented ginseng extracts by gas chromatography mass spectrometry. J. Ginseng Res. 2018, 42, 57-67. [CrossRef]

19. Chemat, F.; Rombaut, N.; Sicaire, A.G.; Meullemiestre, A.; Fabiano-Tixier, A.S.; Abert-Vian, M. Ultrasound assisted extraction of food and natural products. Mechanisms, techniques, combinations, protocols and applications. A review. Ultrason. Sonochem. 2017, 34, 540-560. [CrossRef]

20. Kim, J.Y.; Kim, H.Y.; Jeon, J.Y.; Kim, D.M.; Zhou, Y.; Lee, J.S.; Lee, H.; Choi, H.K. Effects of coronatine elicitation on growth and metabolic profiles of Lemna paucicostata culture. PLoS ONE 2017, 12, e0187622. [CrossRef]

21. Matyash, V.; Liebisch, G.; Kurzchalia, T.V.; Shevchenko, A.; Schwudke, D. Lipid extraction by methyl-tert-butyl ether for high-throughput lipidomics. J. Lipid Res. 2008, 49, 1137-1146. [CrossRef]

22. Kim, S.H.; Lim, S.R.; Hong, S.J.; Cho, B.K.; Lee, H.; Lee, C.G.; Choi, H.K. Effect of ethephon as an ethylene-releasing compound on the metabolic profile of Chlorella vulgaris. J. Agric. Food Chem. 2016, 64, 4807-4816. [CrossRef] [PubMed]

23. Kind, T.; Liu, K.H.; Lee, D.Y.; DeFelice, B.; Meissen, J.K.; Fiehn, O. LipidBlast in silico tandem mass spectrometry database for lipid identification. Nat. Methods 2013, 10, 755-758. [CrossRef] [PubMed]

24. Available online: http://www.lipidmaps.org/ (accessed on 24 October 2018).

25. Bohoyo-Gil, D.; Dominguez-Valhondo, D.; Garcia-Parra, J.; González-Gómez, D. UHPLC as a suitable methodology for the analysis of carotenoids in food matrix. Eur. Food Res. Technol. 2012, 235, 1055-1061. [CrossRef]

26. Kim, S.H.; Liu, K.H.; Lee, S.Y.; Hong, S.J.; Cho, B.K.; Lee, H.; Lee, C.G.; Choi, H.K. Effects of light intensity and nitrogen starvation on glycerolipid, glycerophospholipid, and carotenoid composition in Dunaliella tertiolecta culture. PLoS ONE 2013, 8, e72415. [CrossRef]

27. Available online: http://www.metaboanalyst.ca (accessed on 24 October 2018).

28. Desjardins, M.-L.; Roy, D.; Goulet, J. Growth of bifidobacteria and their enzyme profiles. J. Dairy Sci. 1990, 73, 299-307. [CrossRef]

29. Premi, L.; Sandine, W.E.; Elliker, P.R. Lactose-hydrolyzing enzymes of Lactobacillus species. Appl. Microbiol. 1972, 24, 51-57. [CrossRef]

30. Caspritz, G.; Radler, F. Malolactic enzyme of Lactobacillus plantarum. Purification, properties, and distribution among bacteria. J. Biol. Chem. 1983, 258, 4907-4910.

31. Pham, P.L.; Dupont, I.; Roy, D.; Lapointe, G.; Cerning, J. Production of exopolysaccharide by Lactobacillus rhamnosus $\mathrm{R}$ and analysis of its enzymatic degradation during prolonged fermentation. Appl. Environ. Microbiol. 2000, 66, 2302-2310. [CrossRef]

32. Degeest, B.; Janssens, B.; De Vuyst, L. Exopolysaccharide (EPS) biosynthesis by Lactobacillus sakei 0-1: Production kinetics, enzyme activities and EPS yields. J. Appl. Microbiol. 2001, 91, 470-477. [CrossRef]

33. Palasz, A.; Ciez, D. In search of uracil derivatives as bioactive agents. Uracils and fused uracils: Synthesis, biological activity and applications. Eur. J. Med. Chem. 2015, 97, 582-611. [CrossRef]

34. Elli, M.; Zink, R.; Rytz, A.; Reniero, R.; Morelli, L. Iron requirement of Lactobacillus spp. In completely chemically defined growth media. J. Appl. Microbiol. 2000, 88, 695-703. [CrossRef] [PubMed]

35. Das, G.; Patra, J.K.; Lee, S.Y.; Kim, C.; Park, J.G.; Baek, K.H. Analysis of metabolomic profile of fermented Orostachys japonicus A. Berger by capillary electrophoresis time of flight mass spectrometry. PLoS ONE 2017, 12, e0181280. [CrossRef] [PubMed]

36. Ha, E.M. Escherichia coli-derived uracil increases the antibacterial activity and growth rate of Lactobacillus plantarum. J. Microbiol. Biotechnol. 2016, 26, 975-987. [CrossRef]

37. Liu, Y.Y.; Zeng, S.Y.; Leu, Y.L.; Tsai, T.Y. Antihypertensive effect of a combination of uracil and glycerol derived from Lactobacillus plantarum strain TWK10-fermented soy milk. J. Agric. Food Chem. 2015, 63, 7333-7342. [CrossRef] [PubMed]

38. Olson, K.C.; Sun, D.; Chen, G.; Sharma, A.K.; Amin, S.; Ropson, I.J.; Spratt, T.E.; Lazarus, P. Characterization of dibenzo[ $[a, l]$ pyrene-trans-11,12-diol(dibenzo[def,p]chrysene) glucuronidation by UDP-glucuronosyltransferases. Chem. Res. Toxicol. 2011, 24, 1549-1559. [CrossRef] [PubMed] 
39. Kim, S.R.; Jung, Y.R.; An, H.J.; Kim, D.H.; Jang, E.J.; Choi, Y.J.; Moon, K.M.; Park, M.H.; Park, C.H.; Chung, K.W.; et al. Anti-wrinkle and anti-inflammatory effects of active garlic components and the inhibition of MMPs via NF-kB signaling. PLoS ONE 2013, 8, e73877.

40. Zimin, Y.S.; Borisova, N.; Timerbaeva, G.; Gimadieva, A.; Mustafin, A. Preparation, toxicity, and anti-inflammatory activity of complexes of uracil derivatives with polyfunctional acids. Pharm. Chem. J. 2017, 50, 649-653. [CrossRef]

41. Kim, J.-E.; Chae, C.S.; Kim, G.-C.; Hwang, W.; Hwang, J.-S.; Hwang, S.-M.; Kim, Y.; Ahn, Y.-T.; Park, S.-G.; Jun, C.-D. Lactobacillus helveticus suppresses experimental rheumatoid arthritis by reducing inflammatory T cell responses. J. Funct. Foods 2015, 13, 350-362. [CrossRef]

42. Joo, H.M.; Kim, K.A.; Myoung, K.S.; Ahn, Y.T.; Lee, J.H.; Huh, C.S.; Han, M.J.; Kim, D.H. Lactobacillus helveticus HY7801 ameliorates vulvovaginal candidiasis in mice by inhibiting fungal growth and NF- $\mathrm{KB}$ activation. Int. Immunopharmacol. 2012, 14, 39-46. [CrossRef]

43. Hong, Y.S.; Ahn, Y.T.; Park, J.C.; Lee, J.H.; Lee, H.; Huh, C.S.; Kim, D.H.; Ryu, D.H.; Hwang, G.S. ${ }^{1}$ H NMR-based metabonomic assessment of probiotic effects in a colitis mouse model. Arch. Pharm. Res. 2010, 33, 1091-1101. [CrossRef]

44. Song, H.; Lee, S.Y. Production of succinic acid by bacterial fermentation. Enzyme Microb. Technol. 2006, 39, 352-361. [CrossRef]

45. Zeikus, J.; Jain, M.; Elankovan, P. Biotechnology of succinic acid production and markets for derived industrial products. Appl. Microbiol. Biotechnol. 1999, 51, 545-552. [CrossRef]

46. Dudley, E.G.; Steele, J.L. Succinate production and citrate catabolism by Cheddar cheese nonstarter lactobacilli. J. Appl. Microbiol. 2005, 98, 14-23. [CrossRef]

47. Hillier, A.J. The metabolism of $\left[{ }^{14} \mathrm{C}\right]$ bicarbonate by Streptococcus lactis: The synthesis of succinic acid. J. Dairy Res. 1978, 45, 423-431. [CrossRef] [PubMed]

48. Van der Meulen, R.; Adriany, T.; Verbrugghe, K.; De Vuyst, L. Kinetic analysis of bifidobacterial metabolism reveals a minor role for succinic acid in the regeneration of $\mathrm{NAD}^{+}$through its growth-associated production. Appl. Environ. Microbiol. 2006, 72, 5204-5210. [CrossRef]

49. Giorgi-Coll, S.; Amaral, A.I.; Hutchinson, P.J.A.; Kotter, M.R.; Carpenter, K.L.H. Succinate supplementation improves metabolic performance of mixed glial cell cultures with mitochondrial dysfunction. Sci. Rep. 2017, 7, 1003. [CrossRef]

50. Jalloh, I.; Helmy, A.; Howe, D.J.; Shannon, R.J.; Grice, P.; Mason, A.; Gallagher, C.N.; Stovell, M.G.; van der Heide, S.; Murphy, M.P.; et al. Focally perfused succinate potentiates brain metabolism in head injury patients. J. Cereb. Blood Flow Metab. 2017, 37, 2626-2638. [CrossRef]

51. Iplik, E.S.; Catmakas, T.; Cakmakoglu, B. A new target for the treatment of endometrium cancer by succinic acid. Cell Mol. Biol. 2018, 64, 60-63. [CrossRef]

52. Zarubina, I.V.; Lukk, M.V.; Shabanov, P.D. Antihypoxic and antioxidant effects of exogenous succinic acid and aminothiol succinate-containing antihypoxants. Bull. Exp. Biol. Med. 2012, 153, 336-339. [CrossRef]

53. Carman, G.M.; Henry, S.A. Phosphatidic acid plays a central role in the transcriptional regulation of glycerophospholipid synthesis in Saccharomyces cerevisiae. J. Biol. Chem. 2007, 282, 37293-37297. [CrossRef]

54. Henry, S.A.; Kohlwein, S.D.; Carman, G.M. Metabolism and regulation of glycerolipids in the yeast Saccharomyces cerevisiae. Genetics 2012, 190, 317-349. [CrossRef] [PubMed]

55. Chi, Z.; Kohlwein, S.; Paltauf, F. Role of phosphatidylinositol (PI) in ethanol production and ethanol tolerance by a high ethanol producing yeast. J. Ind. Microbiol. Biotechnol. 1999, 22, 58-63. [CrossRef]

56. Holub, B.J. The nutritional significance, metabolism, and function of myo-inositol and phosphatidylinositol in health and disease. Adv. Nutr. Res. 1982, 4, 107-141. [PubMed]

57. Küllenberg, D.; Taylor, L.A.; Schneider, M.; Massing, U. Health effects of dietary phospholipids. Lipids Health Dis. 2012, 11, 3. [CrossRef] [PubMed]

58. Burgess, J.W.; Neville, T.A.; Rouillard, P.; Harder, Z.; Beanlands, D.S.; Sparks, D.L. Phosphatidylinositol increases HDL-C levels in humans. J. Lipid Res. 2005, 46, 350-355. [CrossRef] [PubMed]

59. Brunton, J.A.; Baldwin, M.P.; Hanna, R.A.; Bertolo, R.F. Proline supplementation to parenteral nutrition results in greater rates of protein synthesis in the muscle, skin, and small intestine in neonatal Yucatan miniature piglets. J. Nutr. 2012, 142, 1004-1008. [CrossRef]

60. Li, P.; Wu, G. Roles of dietary glycine, proline, and hydroxyproline in collagen synthesis and animal growth. Amino Acids 2018, 50, 29-38. [CrossRef] 
61. Barbul, A. Proline precursors to sustain mammalian collagen synthesis. J. Nutr. 2008, 138, 2021s-2024s. [CrossRef]

62. Liang, X.; Dickman, M.B.; Becker, D.F. Proline biosynthesis is required for endoplasmic reticulum stress tolerance in Saccharomyces cerevisiae. J. Biol. Chem. 2014, 289, 27794-27806. [CrossRef]

63. Hayat, S.; Hayat, Q.; Alyemeni, M.N.; Wani, A.S.; Pichtel, J.; Ahmad, A. Role of proline under changing environments: A review. Plant Signal. Behav. 2012, 7, 1456-1466. [CrossRef]

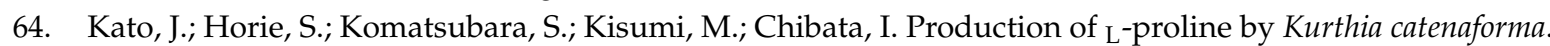
Appl. Microbiol. 1968, 16, 1200-1206. [CrossRef] [PubMed]

65. Prockop, D.J.; Juva, K. Synthesis of hydroxyproline in vitro by the hydroxylation of proline in a precursor of collagen. Proc. Natl. Acad. Sci. USA 1965, 53, 661-668. [CrossRef] [PubMed]

66. Ji, Y.; Guo, Q.; Yin, Y.; Blachier, F.; Kong, X. Dietary proline supplementation alters colonic luminal microbiota and bacterial metabolite composition between days 45 and 70 of pregnancy in Huanjiang mini-pigs. J. Anim. Sci. Biotechnol. 2018, 9, 18. [CrossRef] [PubMed]

67. Ivanov, K.; Stoimenova, A.; Obreshkova, D.; Saso, L. Biotechnology in the production of pharmaceutical industry ingredients: Amino acids. Biotechnol. Biotechnol. Equip. 2013, 27, 3620-3626. [CrossRef]

68. Lee, K.; Kim, H.-J.; Park, S.-K. Amino acids analysis during lactic acid fermentation by single strain cultures of lactobacilli and mixed culture starter made from them. Afr. J. Biotechnol. 2014, 13, 2867-2873.

69. Bannai, M.; Kawai, N.; Ono, K.; Nakahara, K.; Murakami, N. The effects of glycine on subjective daytime performance in partially sleep-restricted healthy volunteers. Front Neurol. 2012, 3, 61. [CrossRef]

70. Rose, M.L.; Madren, J.; Bunzendahl, H.; Thurman, R.G. Dietary glycine inhibits the growth of B16 melanoma tumors in mice. Carcinogenesis 1999, 20, 793-798. [CrossRef]

71. Garrido-Fernandez, J.; Maldonado-Barragan, A.; Caballero-Guerrero, B.; Hornero-Mendez, D.; Ruiz-Barba, J.L. Carotenoid production in Lactobacillus plantarum. Int. J. Food Microbiol. 2010, 140, 34-39. [CrossRef]

72. Arab, L.; Steck, S. Lycopene and cardiovascular disease. Am. J. Clin. Nutr. 2000, 71, 1691S-1695S. [CrossRef]

73. Bartkiene, E.; Vidmantiene, D.; Juodeikiene, G.; Viskelis, P.; Urbonaviciene, D. Lactic acid fermentation of tomato: Effects on cis/trans lycopene isomer ratio, $\beta$-carotene mass fraction and formation of L (+)-and D (-)-lactic acid. Food Technol. Biotech. 2013, 51, 471.

74. Breithaupt, D.E.; Schwack, W.; Wolf, G.; Hammes, W.P. Characterization of the triterpenoid 4, 4'-diaponeurosporene and its isomers in food-associated bacteria. Eur. Food Res. Technol. 2001, 213, 231-233. [CrossRef]

75. Sanchez-Contreras, A.; Jimenez, M.; Sanchez, S. Bioconversion of lutein to products with aroma. Appl. Microbiol. Biotechnol. 2000, 54, 528-534. [CrossRef] [PubMed]

76. Lee, H.; Ahn, Y.T.; Park, S.H.; Park, D.Y.; Jin, Y.W.; Kim, C.S.; Sung, S.H.; Huh, C.S.; Kim, D.H. Lactobacillus plantarum HY7712 protects against the impairment of NK-cell activity caused by whole-body $\gamma$-irradiation in mice. J. Microbiol. Biotechnol. 2014, 24, 127-131. [CrossRef] [PubMed]

77. Jang, S.E.; Joh, E.H.; Lee, H.Y.; Ahn, Y.T.; Lee, J.H.; Huh, C.S.; Han, M.J.; Kim, D.H. Lactobacillus plantarum HY7712 ameliorates cyclophosphamide-induced immunosuppression in mice. J. Microbiol. Biotechnol. 2013, 23, 414-421. [CrossRef]

(C) 2020 by the authors. Licensee MDPI, Basel, Switzerland. This article is an open access article distributed under the terms and conditions of the Creative Commons Attribution (CC BY) license (http://creativecommons.org/licenses/by/4.0/). 